\title{
Planar Curves out of Their Curvatures in $R^{2}$
}

\author{
Talat Alkhouli \\ Applied Science Department, Aqaba College, \\ Al Balqa Applied University, Aqaba, Jordan
}

doi: 10.19044/esj.2016.v12n36p132 URL:http://dx.doi.org/10.19044/esj.2016.v12n36p132

\begin{abstract}
This research aims to introduce some of the main ideas of differential geometry. The research deals with the main concepts needed to understand this work. In this research properties of curves in $R^{2}$ are studied. The research is built on using the curvature of a curve in $R^{2}$ to derive a parametric formula for the velocity and acceleration. Also the geometry of focal points has been discussed. Examples are built to support the aim of this research.
\end{abstract}

Keywords: Curvature, Frenet Formulas, focal point, planner curves.

\section{Introduction}

The main content of this research is some differential geometry concerning curves in $R^{2}$. a brief description of curves in $R^{2}$ is introduced. The geometry of curves in $R^{2}$ is described using Frenet formulas. The concept of a focal point is a base within this research, so definitions and theorems concerning focal points are included. As this research is about geometry of curves, the curvature of a curve in $R^{2}$ is studied in the field of focal points.

Differential geometry is a mathematical field which allows the study of geometrical concepts using calculus. Thus, calculus is used to explain how to extract planar curves out of their curvatures, an idea which supports the content of this research. Examples are built to support the aim of this research. Other geometrical concepts are included.

Curves in $R^{2}$

Definition 1.1. A curve $C$ in $R^{2}$ is a differentiable function $\vec{f}: \mathrm{I} \rightarrow R^{2}$ where I is an (open) interval.

Thus, if $t$ is a parameter of $\vec{f}, t \in \mathrm{I}$, then we write $\vec{f}$ as

$$
\vec{f}(t)=\left(f_{1}(t), f_{2}(t)\right)
$$

where $f_{1}$ and $f_{2}$ are differentiable real valued functions defined on I. 
We say that $\vec{f}$ is a parametrization of $C$ or $C$ is the trace of $\vec{f}$ on I .

Definition 1.2. A curve $C$ in $\mathrm{R}^{2}$ with parametrization $\vec{f}: \mathrm{I} \rightarrow R^{2}$ is regular provided that $\vec{f}^{\prime}(t) \neq \overrightarrow{0}$ for all $t \in \mathrm{I}$. If $\vec{f}^{\prime}\left(t_{1}\right)=\overrightarrow{0}$ for some $t_{1} \in \mathrm{I}$, then $\vec{f}\left(t_{1}\right)$ is a singular point of $C$.

Definition 1.3. Let $\vec{f}: \mathrm{I} \rightarrow R^{2}$ be a curve in $\mathrm{R}^{2}$. Let $\delta: J \rightarrow \mathrm{I}$ be a differentiable real valued function where $J$ is also an interval. Then $\vec{w}=\vec{f}(\delta): J \rightarrow R^{2}$ is a reparametrization of $\vec{f}$ by $\delta$.

Definition 1.4. The reparametrization $\vec{w}$ of $\vec{f}$ is orientation preserving if $\delta^{\prime}>0$ on $J$ and orientation reversing if $\delta^{\prime}<0$ on $J$.

Now we take $\mathrm{I}=[a, b]$ and define the arclength along $C$ by

$$
s(t)=\int_{a}^{t}\left\|\vec{f}^{\prime}(u)\right\| d u
$$

where $\left\|\vec{f}^{\prime}(t)\right\|$ is the norm of $\vec{f}^{\prime}(t)$, usually called the speed of $\vec{f}$. If $C$ is a regular curve, then $\left\|\vec{f}^{\prime}(t)\right\|>0$ for all $t \in \mathrm{I}$ and so $s$ is a strictly increasing function of $t$ which has an inverse. That is, Equation (1) can be solved for $s$ and then $C$ has a reparametrization by arclength.

Proposition 1.5. Let $\vec{w}$ be an arclength reparametrization of $\vec{f}$. Then $\vec{w}$ has unit speed.

Proof: Observe that $\vec{w}$ is defined by $\vec{w}(s)=\vec{f}(t(s))$. Then differentiate with respect to $s$ to get

$$
\left\|\frac{d \vec{w}}{d s}\right\|=\left\|\frac{d \vec{f}}{d t} \frac{d t}{d s}\right\|=\mid \frac{d t}{d s}\left\|\frac{d \vec{f}}{d t}\right\|=\frac{1}{\left\|f^{\prime}\right\|}\left\|f^{\prime}\right\|=1 . \diamond
$$

Reparametrizing a regular curve by acrlength (also called unit speed reparametrization) simplifies calculations regarding geometry of curves.

\section{Curvature of a curve in $R^{2}$}

Now take $\vec{w}$ as a unit speed curve, and then $\vec{w}^{\prime \prime} \perp \vec{w}^{\prime}$. For, $\left\|\vec{w}^{\prime}\right\|=1 \Rightarrow \vec{w}^{\prime} \cdot \vec{w}^{\prime}=1 \Rightarrow \vec{w}^{\prime} \cdot \vec{w}^{\prime \prime}=0$.

If $\overrightarrow{\mathrm{T}}(s)$ is the unit tangent of $\vec{w}$ at $s$, then $\overrightarrow{\mathrm{T}}=\vec{w}^{\prime}$.

Also

$\overrightarrow{\mathrm{T}}^{\prime}=\vec{w}^{\prime \prime}$.

Definition 2.1. The curvature of a unit speed curve $\vec{w}$ is the function $\kappa(s)=\left\|\overrightarrow{\mathrm{T}}^{\prime}(s)\right\|$ where $\overrightarrow{\mathrm{T}}$ is the unit tangent of $\vec{w}$.

Now let $\overrightarrow{\mathrm{N}}(s)$ be the unit normal of $\vec{w}$ at $s$, 
then

$$
\overrightarrow{\mathrm{T}}^{\prime}(s)=\kappa(s) \overrightarrow{\mathrm{N}}(s)
$$

The orthonormal set of the vectors $\{\overrightarrow{\mathrm{T}}, \overrightarrow{\mathrm{N}}\}$ is called Frenet frame. So $\overrightarrow{\mathrm{N}}^{\prime}$ can be written in terms of $\overrightarrow{\mathrm{T}}$ and $\overrightarrow{\mathrm{N}}$. In fact, if $\overrightarrow{\mathrm{N}}^{\prime}=a \overrightarrow{\mathrm{T}}+b \overrightarrow{\mathrm{N}}$, then

$b=\overrightarrow{\mathrm{N}}^{\prime} \cdot \overrightarrow{\mathrm{N}}=0$

and by Equation (2),

$a=\overrightarrow{\mathrm{N}}^{\prime} \cdot \overrightarrow{\mathrm{T}}=-\overrightarrow{\mathrm{N}} \cdot \overrightarrow{\mathrm{T}}^{\prime}=-\overrightarrow{\mathrm{N}} \cdot \kappa \overrightarrow{\mathrm{N}}=-\kappa$.

Hence

$$
\overrightarrow{\mathrm{N}}^{\prime}(s)=-\kappa(s) \overrightarrow{\mathrm{T}}(s)
$$

Equations (2) and (3) are called Frenet formulas.

Now let $\vec{f}$ be an arbitrary regular curve with parameter $t$ and unit speed reparametrization $\vec{w}(s)$. Let $v(t)=\left\|\vec{f}^{\prime}(t)\right\|$.

Theorem 2.2. Frenet formulas of $\vec{f}$ are

$$
\begin{aligned}
& \overrightarrow{\mathrm{T}}^{\prime}(t)=\kappa(t) v(t) \overrightarrow{\mathrm{N}}(t) \\
& \overrightarrow{\mathrm{N}}^{\prime}(t)=-\kappa(t) v(t) \overrightarrow{\mathrm{T}}(t)
\end{aligned}
$$

Proof: It is clear that there is a correspondence between points on $\vec{f}$ and $\vec{w}$ in a way that $\exists t$ and $s$ such that

$$
\vec{f}(t)=\vec{w}(s), \quad \overrightarrow{\mathrm{T}}_{\bar{f}}(t)=\overrightarrow{\mathrm{T}}_{\bar{w}}(s), \quad \overrightarrow{\mathrm{N}}_{\bar{f}}(t)=\overrightarrow{\mathrm{N}}_{\bar{w}}(s) \quad \text { and }
$$
$\kappa_{\bar{f}}(t)=\kappa_{\bar{w}}(s)$.

Thus, Equation (2) implies that

$\overrightarrow{\mathrm{T}}^{\prime}(t)=\frac{d \overrightarrow{\mathrm{T}}}{d s} \frac{d s}{d t}=\kappa(s) \overrightarrow{\mathrm{N}}(s) \frac{d s}{d t}=\kappa(t) v(t) \overrightarrow{\mathrm{N}}(t)$.

Also Equation (3) implies that

$$
\overrightarrow{\mathrm{N}}^{\prime}(t)=\frac{d \overrightarrow{\mathrm{N}}}{d s} \frac{d s}{d t}=-\kappa(s) \overrightarrow{\mathrm{T}}(s) \frac{d s}{d t}=-\kappa(t) v(t) \vec{T}(t) . \diamond
$$

Theorem 2.3. The velocity and acceleration vectors of $\vec{f}$ at $t$ are respectively

$$
\begin{aligned}
\vec{f}^{\prime}(t) & =v(t) \overrightarrow{\mathrm{T}}(t) \\
\vec{f}^{\prime \prime}(t) & =v^{\prime}(t) \overrightarrow{\mathrm{T}}(t)+\kappa(t) v^{2}(t) \overrightarrow{\mathrm{N}}(t)
\end{aligned}
$$

Proof: The unit tangent of $\vec{f}$ at $t$ is $\overrightarrow{\mathrm{T}}(t)=\frac{\vec{f}^{\prime}(t)}{\left\|\vec{f}^{\prime}(t)\right\|}$,

and so

$$
\vec{f}^{\prime}(t)=\left\|f^{\prime}(t)\right\| \overrightarrow{\mathrm{T}}(t)=v(t) \overrightarrow{\mathrm{T}}(t),
$$

and so Equation (6) is derived. 
Now differentiate Equation (6) with respect to $t$, then use Equation (4) to get Equation (7). $\diamond$

Theorem 2.4. Let $\vec{f}$ be an arbitrary regular curve. Then the curvature of $\vec{f}$ is given by

$$
\kappa(t)=\frac{\left\|\vec{f}^{\prime}(t) \times \vec{f}^{\prime \prime}(t)\right\|}{\left\|\vec{f}^{\prime}(t)\right\|^{3}}
$$

Proof: By Equations (6) and (7), we have

$\vec{f}^{\prime} \times \vec{f}^{\prime \prime}=v \overrightarrow{\mathrm{T}} \times\left(v^{\prime} \overrightarrow{\mathrm{T}}+\kappa v^{2} \overrightarrow{\mathrm{N}}\right)=\kappa v^{3} \overrightarrow{\mathrm{T}} \times \overrightarrow{\mathrm{N}}$.

Thus,

$\left\|\vec{f}^{\prime} \times \vec{f}^{\prime \prime}\right\|=\kappa v^{3}\|\overrightarrow{\mathrm{T}} \times \overrightarrow{\mathrm{N}}\|=\kappa v^{3}$.

Hence Equation (8) follows. $\diamond$

Definition 2.5. The total curvature of a regular curve $\vec{f}$ on I is

$$
\mathrm{K}=\int_{\mathrm{I}} v(t) \kappa(t) d t
$$

The focal curve of a curve in $R^{2}$

Definition 3.1. The focal curve of a regular curve $\vec{f}$ is the curve $\vec{g}=\vec{f}+\frac{1}{\kappa} \overrightarrow{\mathrm{N}}$.

Similarly, the focal point with base $\vec{f}(t)$ is the point $\vec{g}(t)=\vec{f}(t)+\frac{1}{\kappa(t)} \overrightarrow{\mathrm{N}}(t)$.

Now let $\Lambda_{\vec{p}}(t)=\|\vec{f}(t)-\vec{p}\|^{2}$ be the distance function whose domain is the curve $C$ with parametrization $\vec{f}$.

Theorem 3.2. The point $\vec{p}$ is a focal point of $C$ with base $\vec{f}(t)$ iff $\Lambda_{\vec{p}}^{\prime}(t)=0$ and $\Lambda_{\vec{p}}^{\prime \prime}(t)=0$.

Proof: Recall that $\Lambda_{\vec{p}}(t)=\|\vec{f}(t)-\vec{p}\|^{2}=(\vec{f}(t)-\vec{p}) \cdot(\vec{f}(t)-\vec{p})$.

Thus,

$\Lambda_{\vec{p}}^{\prime}(t)=2(\vec{f}(t)-\vec{p}) \cdot \vec{f}^{\prime}(t)$

And $\quad \Lambda_{\vec{p}}^{\prime \prime}(t)=2(\vec{f}(t)-\vec{p}) \cdot \vec{f}^{\prime \prime}(t)+2 \vec{f}^{\prime}(t) \cdot \vec{f}^{\prime}(t)$.

If $\vec{p}$ is a focal point of $C$ with base $\vec{f}(t)$, then $\vec{p}=\vec{f}(t)+\frac{1}{\kappa(t)} \overrightarrow{\mathrm{N}}(t)$. Thus, 


$$
\begin{aligned}
\Lambda_{\vec{p}}^{\prime}(t)=2(\vec{f}(t)-\vec{f}(t) & \left.-\frac{1}{\kappa(t)} \overrightarrow{\mathrm{N}}(t)\right) \cdot \vec{f}^{\prime}(t) \\
& =\frac{-2}{\kappa(t)} \overrightarrow{\mathrm{N}}(t) \cdot \vec{f}^{\prime}(t)=0 .
\end{aligned}
$$

Also

$$
\Lambda_{\vec{p}}^{\prime \prime}(t)=2\left(\vec{f}(t)-\vec{f}(t)-\frac{1}{\kappa(t)} \overrightarrow{\mathrm{N}}(t)\right) \cdot \vec{f}^{\prime \prime}(t)+2 \vec{f}^{\prime}(t) \cdot \vec{f}^{\prime}(t)
$$

$$
\begin{aligned}
& =\frac{2}{\kappa(t)} \overrightarrow{\mathrm{N}}^{\prime}(t) \cdot \vec{f}^{\prime}(t)+2 \vec{f}^{\prime}(t) \cdot \vec{f}^{\prime}(t) \\
& =-2 \vec{f}^{\prime}(t) \cdot \vec{f}^{\prime}(t)+2 \vec{f}^{\prime}(t) \cdot \vec{f}^{\prime}(t)=0 .
\end{aligned}
$$

Conversely, the equation $\Lambda_{\vec{p}}^{\prime}(t)=0$ implies that $\vec{f}(t)-\vec{p}=m \overrightarrow{\mathrm{N}}$. Now put $\vec{p}=\vec{f}(t)-m \overrightarrow{\mathrm{N}}$ in the equation $\Lambda_{\vec{p}}^{\prime \prime}(t)=0$ to get

$m \overrightarrow{\mathrm{N}} \cdot \vec{f}^{\prime \prime}(t)+\vec{f}^{\prime}(t) \cdot \vec{f}^{\prime}(t)=0$,

which is equivalent to

$-m \overrightarrow{\mathrm{N}}^{\prime} \cdot \vec{f}^{\prime}(t)+\vec{f}^{\prime}(t) \cdot \vec{f}^{\prime}(t)=0$

or

$m \kappa(t) \vec{f}^{\prime}(t) \cdot \vec{f}^{\prime}(t)+\vec{f}^{\prime}(t) \cdot \vec{f}^{\prime}(t)=0$.

Thus, $\quad m=-\frac{1}{\kappa(t)}$. So $\vec{p}=\vec{f}(t)+\frac{1}{\kappa(t)} \overrightarrow{\mathrm{N}}(t)$, i.e. $\vec{p}$ is a focal point of $C$ with base $\vec{f}(t) . \diamond$

Example 3.3. Let $\vec{f}(t)=\left(t, t^{2}\right)$. Then

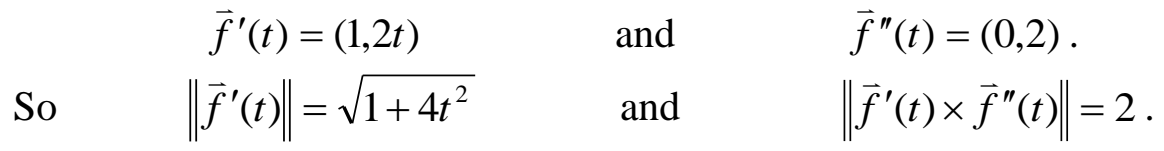

Thus,

$$
\kappa(t)=\frac{2}{\left(\sqrt{1+4 t^{2}}\right)^{3}} .
$$

By solving the equation

$$
(0,2)=\frac{4 t}{\sqrt{1+4 t^{2}}} \frac{(1,2 t)}{\sqrt{1+4 t^{2}}}+\frac{2}{\left(\sqrt{\left.1+4 t^{2}\right)^{3}}\right.}\left(1+4 t^{2}\right) \overrightarrow{\mathrm{N}}(t)
$$

for $\overrightarrow{\mathrm{N}}(t)$, we get

$$
\overrightarrow{\mathrm{N}}(t)=\frac{(-2 t, 1)}{\sqrt{1+4 t^{2}}} \text {. }
$$

Thus, 


$$
\begin{aligned}
\vec{g}(t)=\left(t, t^{2}\right)+\frac{\left(\sqrt{1+4 t^{2}}\right)^{3}}{2} & \frac{(-2 t, 1)}{\left.\sqrt{1+4 t^{2}}\right)} \\
= & \left(-4 t^{3}, \frac{1}{2}+3 t^{2}\right) .
\end{aligned}
$$

Now $\quad \Lambda_{\bar{p}}(t)=(t-a)^{2}+\left(t^{2}-b\right)^{2}$ with $\vec{p}=(a, b)$. So

$$
\Lambda_{\bar{p}}^{\prime}(t)=(2-4 b) t+4 t^{3}-2 a
$$

and

$\Lambda_{\vec{p}}^{\prime \prime}(t)=2-4 b+12 t^{2}$.

Equating the last two equations to zero, we get

$$
b=1 / 2+3 t^{2} \quad \text { and } \quad a=-4 t^{3} \text {. }
$$

Example 3.4. Consider the curve defined by :

$\vec{f}(t)=(9 \cos t+\cos t \cos 3 t+3 \sin t \sin 3 t, 9 \sin t+\sin t \cos 3 t-3 \cos t \sin 3 t)$ Then

$$
\vec{f}^{\prime}(t)=(9-8 \cos 3 t)(-\sin t, \cos t)
$$

and

$$
\vec{f}^{\prime \prime}(t)=(9-8 \cos 3 t)(-\cos t,-\sin t)+24 \sin 3 t(-\sin t, \cos t) .
$$

So

$$
\left\|\vec{f}^{\prime}(t)\right\|=9-8 \cos 3 t \quad \text { and } \quad\left\|\vec{f}^{\prime}(t) \times \vec{f}^{\prime \prime}(t)\right\|=(9-8 \cos 3 t)^{2} .
$$

Thus,

$$
\kappa(t)=\frac{1}{9-8 \cos 3 t} .
$$

By solving the equation

$$
\vec{f}^{\prime \prime}(t)=24 \sin 3 t(-\sin t, \cos t)+\frac{1}{9-8 \cos 3 t}(9-8 \cos 3 t)^{2} \overrightarrow{\mathrm{N}}(t)
$$

for $\overrightarrow{\mathrm{N}}(t)$, we get $\overrightarrow{\mathrm{N}}(t)=(-\cos t,-\sin t)$.

Thus,

$$
g(t)=(9 \cos t \cos 3 t+3 \sin t \sin 3 t, 9 \sin t \cos 3 t-3 \cos t \sin 3 t)
$$

Let $\vec{p}=(a, b)$. Then

$$
\begin{aligned}
& \Lambda_{\bar{p}}(t)=(9 \cos t+\cos t \cos 3 t+3 \sin t \sin 3 t-a)^{2}+(9 \sin t+\sin t \cos 3 t-3 \cos t \sin 3 t-b)^{2} \\
& \text {. So } \Lambda_{\vec{p}}^{\prime}(t)=2(9-8 \cos t)(-3 \sin 3 t+a \sin t-b \cos t),
\end{aligned}
$$


$\Lambda_{\bar{p}}^{\prime \prime}(t)=2(9-8 \cos 3 t)(-9 \cos 3 t+a \cos t+b \sin t)+48 \sin 3 t(-3 \sin 3 t+a \sin t-b \cos t)$

Equating the last two equations to zero, we get

$-3 \sin 3 t+a \sin t-b \cos t=0$

and

$-9 \cos 3 t+a \cos t+b \sin t=0$.

Solving the last two equations together, we get

$a=9 \cos t \cos 3 t+3 \sin t \sin 3 t \quad$ and $\quad b=9 \sin t \cos 3 t-3 \cos t \sin 3 t$.

\section{Planar curves out of their curvatures}

Let $\vec{f}$ be a planar curve with parameter $s$, the arclength. Let $\phi(s)$ be the slope angle of the tangent line at $\vec{f}(s)$. Then the unit tangent of $\vec{f}$ at $s$ is defined by $\tau(s)=(\cos \phi(s), \sin \phi(s))$.

Now $k(s)=\left\|\frac{d \tau}{d s}\right\|=\frac{d \phi}{d s}$. Thus,

$$
\phi(s)=\int_{0}^{s} k(s) d s
$$

Now if $\vec{f}(s)=(x(s), y(s))$, then

$$
x(s)=\int_{0}^{s} \cos \phi(s) d s
$$

and

$$
y(s)=\int_{0}^{s} \sin \phi(s) d s
$$

Example 4.1. Let $k(s)=\frac{1}{3}$ with $\vec{f}(0)=(0,0)$. Then by Equation (9),

$$
\phi(s)=\int_{0}^{s} k(s) d s=\frac{1}{3} s .
$$

By Equation (10), we have

$$
x(s)=\int \cos \frac{1}{3} s d s=3 \sin \frac{1}{3} s+c_{1} .
$$

But $x(0)=0$, and so $x(s)=3 \sin \frac{1}{3} s$. Also by Equation (11), we have 
$y(s)=\int \sin \frac{1}{3} s d s=-3 \cos \frac{1}{3} s+c_{2}$.

But $y(0)=0$, and so $y(s)=-3 \cos \frac{1}{3} s+3$.

So $\vec{f}(s)=\left(3 \sin \frac{1}{3} s,-3 \cos \frac{1}{3} s+3\right)$, which is a circle of radius 3 .

Example 4.2. Let $k(s)=\frac{1}{s^{2}+1}$ with $\vec{f}(0)=(0,0)$. Then by Equation (9), $\phi(s)=\int_{0}^{s} k(s) d s=\int_{0}^{s} \frac{1}{s^{2}+1} d s=\tan ^{-1} s$.

Now by Equation (10), we have

$$
\begin{aligned}
x(s) & =\int \cos \left(\tan ^{-1} s\right) d s \\
& =\int \frac{1}{\sqrt{s^{2}+1}} d s=\sinh ^{-1} s+c_{1} .
\end{aligned}
$$

But $x(0)=0$, and so $x(s)=\sinh ^{-1} s$. Also by Equation (11), we have

$$
\begin{aligned}
y(s) & =\int \sin \left(\tan ^{-1} s\right) d s . \\
& =\int \frac{s}{\sqrt{s^{2}+1}} d s=\sqrt{s^{2}+1}+c_{2} .
\end{aligned}
$$

But $y(0)=0$, and so $y(s)=\sqrt{s^{2}+1}-1$. So $\vec{f}(s)=\left(\sinh ^{-1} s, \sqrt{s^{2}+1}-1\right)$.

\section{References:}

1. Al-Banawi K, Generating Frames and Normal Holonomy of Transnormal Submanifolds in Euclidean Spaces, $\mathrm{PhD}$ thesis, University of Leeds, UK, 2004.

2. Al-Banawi K, Geometry of Ovals in Terms of the Support Function, Journal of Applied Sciences, 8(2)(2008):383-386.

3. Al-Banawi K, Some Notes on Classical Differential Geometry, Department of Mathematics and Statistics, Mutah University (Course Notes), 2011.

4. Eggleston H, Convexity, Cambridge Tracts in Mathematics, 47, Cambridge University Press, 1958. 
5. Hsiung C, A First Course in Differential Geometry, John Wiley and Sons, 1981.

6. O’Neill B. Elementary Differential Geometry (2 ${ }^{\text {nd }}$ Edition), Academic Press, London, 1997.

7. Struik D, Lectures on Classical Differential Geometry, AddisonWesley, New York, 1950. 